\title{
De la transgression dans les apprentissages : usages et limites
}

Marie BERCHOUD, Université de Bourgogne, France

\section{Introduction}

Ce qu'on nomme couramment la transgression parait être le fait de s'affranchir d'une norme requise, que celle-ci soit familiale ou sociale, le plus souvent individuellement incorporée ; autrement dit, c'est le franchissement de limites inhérentes à la norme, ce qui alors la révèle et peut-être la fragilise. En effet, il n'y a pas une mais des normes, et les normes peuvent venir en conflit. Dans l'iconographie des manuels scolaires représentant les enfants élèves qui apprennent, la première norme est celle du contrôle du corps : être assis, se tenir convenablement, être occupé à une tâche qui est le plus souvent, lire ou apprendre à lire. Ces représentations sont le plus souvent sexuées, la fille étant un peu plus âgée, et surtout bien plus sage que le garçon, et cela depuis un bon siècle (voir exemples en annexe).

La transgression ainsi définie met en question le mode socio-scolaire d'apprentissage, qui vient buter contre les libertés et rigueurs de l'école de la vie. On le voit, par exemple avec Alice au pays des merveilles qui, délaissant l'étude et la lecture (à laquelle son aînée est occupée et qu'elle pourrait partager avec elle), choisit la nature contre la culture symbolisée par le livre, et le corps libre par-delà le corps discipliné par l'étude : elle s'agite, tombe et se retrouve ainsi dans un trou-tunnel qui la mènera aux aventures que l'on sait, soit un apprentissage, mais tout à fait non scolaire, même s'il a traversé les siècles. Et peut-être cet apprentissage en forme d'aventure individuelle fait-il contrepoint aux apprentissages socioscolaires.

Il faut donc se demander si transgresser - mais transgresser quoi, quel ordre ? Et pourquoi, pour quoi faire, être, exister ? - serait une condition pour apprendre : à travers plusieurs cas et situations d'apprentissages en langues vivantes (apprentissages scolaires et pas seulement, cf. Hagège) nous verrons ce qui peut être nommé transgression et sur quelles bases, puis quels usages peuvent être faits de ces «transgressions ». Celles-ci peuvent être le fait de tous les acteurs, qu'ils soient éducateurs ou enseignants, voire par les apprenants concernés eux-mêmes : il faudrait alors imaginer un processus continué d'auto-éducation, de connaissance de soi et d'autonomisation progressive que les approches dites actives ou non conventionnelles s'attachent à développer. Mais peut-on pour autant généraliser la transgression, ce qui conduirait à la constituer en une nouvelle norme, on peut en douter. Il semble bien que transgresser doive demeurer une aventure individuelle avec ses chances et 
ses risques, aventure née d'un mouvement de l'être qui par ce choix se constitue en être humain.

\section{Transgresser pour apprendre?}

Quelle est la part de la norme ou des normes dans les apprentissages ? Et celle de leur transgression ? Et puis : qui transgresse ? Certains élèves, lesquels ? Quant aux enseignants ? Nous venons de voir que la notion de transgression établit un partage entre apprentissage dans le cadre de l'institution scolaire qui contient et contraint les corps (pour ne rien dire encore des esprits), et apprentissage par l'expérience, qui alors engage l'ensemble de l'être humain dans l'aventure.

Transgresser à l'école, ce serait alors aller contre la norme, la mettre en question, tenter d'en atténuer la rigueur : s'agiter, bavarder, la chose est banale, une espèce d'exutoire ; mais aussi poser des questions jugées inadéquates, aller trop vite pour apprendre, choisir des voies singulières. Ici apparaît un autre caractère de la norme, la norme est commune, elle serait la condition d'un enseignement et d'un apprentissage collectif.

Oui mais, qui apprend? C'est l'élève, c'est chacun des enfants ou adolescents. Si l'enseignement est collectif, l'apprentissage ne peut être qu'individuel, du moins s'il est effectif et assorti de résultats. Chaque enseignant a eu à faire avec les diverses stratégies d'évitement des élèves, par exemple, pour ne pas être interrogés. Tout apprentissage s'inscrit donc dans une temporalité de l'ordre de la durée vécue, du rythme personnel, et non du temps scolaire. La question de la transgression convoque donc son corollaire, la question de l'individualisation des apprentissages.

Transgresser pour apprendre, alors, oui, et Philippe Meirieu en est bien d'accord. Voyons comment : du côté des enseignants, il est certain que prendre à part un élève pour réexpliquer, c'est rompre la règle de l'enseignement collectif, tél qu'édicté sous la troisième République ; mais c'est aussi le bon sens et l'attention à autrui, autres règles. Du côté des élèves, il est avéré qu'on n'apprend pas sans motivation, et celle-ci est profondément personnelle, donc chacun, s'il veut apprendre, va être amené à une transgression réinterprétation singulière de la norme, et sans doute alors, s'approprier la norme est-ce quelque peu la transformer, la mettre à sa main, au diapason de son cœur.

\subsection{Formes variées de la transgression : un écart que «je »choisis}

Comment cela? L'expérience vécue comme enseignante, et comme élève, étudiante donne des pistes utiles (Berchoud, Rui et Mallet) : d'abord ne pas perdre le contact avec son soi profond, intime, être à l'écoute ; et de ce fait, devenir capable de dire «non », de faire des 
choix autres que ceux du plus grand nombre ; capable de bouger, et pas seulement dans l'agitation ou le bavardage exutoires, mais dans la séparation temporaire d'avec le groupeclasse ; être ou devenir capable d'affronter des risques, et même d'oser ne pas savoir, d'oser (se) poser des questions car le goût d'apprendre du nouveau, de découvrir contrebalance la crainte du risque. Regardez Alice au pays des Merveilles, elle fait face, même si, à la fin, elle se demande vaguement comment elle a pu faire. Elle l'a fait.

Prenons un exemple actuel ou du moins assez proche de nous - années 2004 et suivantes : une enquête sur les enfants qui apprennent malgré... tout, même au milieu des pires difficultés qui auraient terrassé bien d'autres enfants, montre chez ces enfants qui apprennent quand même l'appropriation de façons de faire et d'être favorables aux apprentissages (Berchoud, 4-11, 57) : leur donner du sens pour soi, savoir apprendre, saisir les règles... avec recul. Car celui ou celle qui a l'expérience d'une réussite, même nonscolaire, en un domaine au moins, se sent dans une sécurité existentielle convenable et dispose de la capacité à communiquer avec autrui et pas seulement ses proches. En clair, cela revient à ne pas se situer totalement dans le collectif.; et à savoir distinguer l'essentiel de l'accessoire. Par exemple, s'agit-il dans cet exercice de souligner les verbes en couleur et de les classer en colonnes selon le temps employé ? Ou de comprendre comment fonctionne la concordance des temps? Elisabeth Bautier (105-118) combien cette distinction est essentielle : les maîtres ont à s'efforcer de mener chaque élève au vrai but de l'exercice ou de l'activité, par-delà les apparences.

\subsection{La transgression : une orientation, des couples d'opposition, une praxis}

Il semble donc y avoir transgression et transgression, transgresser pour / transgresser contre, autrement dit dans le premier cas un projet, et dans le second, une protestation, un refus. Remarquons aussi que la transgression est aujourd'hui valorisée dans les médias, qu'elle corresponde au non à une création, une initiative : « un film transgressif et jouissif » (Les immortels, 2011) ; «un jeu transgressif et jouissif »... «une indifférence active et transgressive » (Bart 138, 139). Que transgressent donc ce film, ce jeu... et pourquoi ? Des usages, un genre cinématographique ? Ou tout simplement une norme sociale qui, du reste, ne leur est pas ou plus imposée ? On peut se le demander, et s'interroger sur l'intérêt d'une transgression « contre » surtout si elle est sans risque, voire une transgression par procuration au cinéma. Catharsis, défoulement ? Peut-être. Le rire fait du bien aussi.

Transgresser «pour»semble en revanche plus porteur de sens et de perspectives : Antigone dans la pièce de Sophocle transgresse la loi de la Cité pour respecter l'Autre loi, supérieure. Pour cela, elle risque sa vie, et la perdra, ou plutôt elle accepte de la donner. Mais 
il s'agit d'un exemple extrême. Retenons que la transgression «pour » a un but, se réfère à une règle autre, et ne vient pas seulement s'attaquer ou s'adosser à la norme pour la contester.

Nous voici donc amenés à structurer plus précisément la notion de transgression, autour de couples d'oppositions qui concourent à tracer le périmètre de la notion et de ses applications :

- $\quad$ Dans / au dehors de la ou les normes requises

- $\quad$ En un espace organisé ou pas

- $\quad$ Avec un corps contraint ou pas

- $\quad$ En groupe / individuellement

- Dans la sécurité / avec des risques

- $\quad$ Selon une sujétion / en autonomie

- $\quad$ À la demande / selon un choix autonome

On le perçoit ici, nous avons besoin du couple d'oppositions, sans lequel la norme serait totalitaire ; toute norme est donc limitée par son contrepoint, son extériorité ; ainsi la notion de transgression est dynamique plutôt que statique, c'est une praxis. De façon plus générale, celle-ci peut être reliée à l'opposition chrétienne entre la lettre et l'esprit, « la lettre tue et l'esprit vivifie » (Paul, Corinthiens II, $3: 6$ ). Cela se traduit dans le droit, par exemple avec la notion d' «ordre manifestement illégal» (article 122-4, al. 2, Code pénal français) auquel un fonctionnaire ou un militaire a le droit de désobéir, et pas forcément en situation d'urgence.

Situons à présent ces couples d'opposition dans un contexte francophone actuel, et interrogeons le Dictionnaire culturel (Le Robert, 2005). La transgression s'y définit comme «un interdit». Il y a oubli de l'élément moteur, commun à progresser / régresser, soit un sujet singulier au profit d'une norme préexistante, rigide, externe et intériorisée, quasisacrée. Un sens positif émerge seulement de l'interrogation de la norme : le «je »sujet, au premier plan, avec d'autres. Alors, s'ensuit-il qu'il y aurait deux types de pédagogies pour apprendre et faire apprendre ? Ou un au-delà de toute pédagogie ? On pense ici aux Libres enfants de Summerhill qui se construisaient eux-mêmes, en relation avec leurs enseignants leurs règles pour vivre en collectivité et apprendre. Il convient en tout cas de s'intéresser aux contextes dans lesquels intervient la transgression. Nous repoussons ou nous reconsidérons les limites, les interdits, les règles et leur respect. Mais selon quel) principe organisateur ? Il semble que soit essentielle la présence d'une autre loi, structurée, et distincte du sujet, de son bon plaisir et de sa toute-puissance. 


\subsection{L'autre loi ? Regard sur quelques apprentissages de langue}

Les enfants bilingues ou plurilingues sont confrontés à une double norme, celle de chaque langue et de ses modes d'apprentissage de référence. C'est pourquoi «les déviances sont chez l'enfant des instruments pour apprendre » selon Claude Hagège (85), qui dit oui au jeu sur le et les langues, même aux limites, mais à condition qu'il soit accompagné, pour parer au danger du semi-linguisme et du mélange des langues (103, 262-262). Plus généralement, il est acquis que l'on peut apprendre (de) la transgression : «On conçoit sans peine l'intérêt didactique d'un apprentissage réglé de la transgression : l'appropriation de la 'parole' passe par la manipulation lucide et 'distanciée' des codes, mais aussi par une implication personnelle à laquelle la composante plus ludique de cette activité n'est sans doute pas étrangère. » (Blaise 90)

Peut-on alors extrapoler des langues, qui, certes, sont très propres à l'individu, aux autres apprentissages ? Autrement dit, peut-on apprendre via la transgression ? Et comment transgresser, à quel moment effectuer ce pas de côté, ce mouvement personnel de mise en accord de soi avec l'objet à apprendre ? Et enfin, transgresser, faire ce pas singulier, pour aller où ? Simplement trouver son rythme, sa marche, son geste, ses façons d'être et de faire ? Apprendre est en tout cas un acte vital et, en tout cas, bien propre à l'espèce humaine, il se produit dans une variété de contextes et situations que nous pourrions approcher pour en savoir un peu plus.

\section{Contextes, objectifs et usages de la transgression dans les apprentissages}

Transgresser pour progresser, choisir un univers et ses lois et/ou l'exercice du droit à exister comme personne individuelle, contre un autre univers et ses lois collectives, nous en sommes là. Détaillons ici cette tension entre collectif et individuel, qui va au-delà de la réflexion sur le poids des contextes, en ce qu'elle considère aussi l'action et les attitudes des personnes entourant celui ou celle qui apprend.

\subsection{De la fiction aux réalités vécues et constatées : aller et retour}

Revenons sur le cas d'Alice au pays des merveilles. Nous avons un contexte, et une situation initiale :

L'ennui d'Alice, la nature, sa sœur aînée qui lit

Une rêverie : en soi-même, et dehors, hors ses cadres

Une rencontre : soi, l'autre, les autres

Un engagement dans l'aventure : intérieure, extérieure 
Une capacité à ne rien tenir pour acquis qu'on ne l'ait vérifié à (se) poser des questions

Et le goût de la découverte.

Alice va s'ouvrir un chemin singulier, le sien, et ce sera une voie de la transformation. Quelle fatigue, au final, mais quelle richesse !

Alice est une fiction, et son intérêt est bel et bien de se constituer en modèle, en voie à suivre et à rêver, mais aussi en balisage du chemin vers l'autonomie, voire l'auto-éducation.

Pour revenir au temps présent, prenons d'autres exemples d'apprentissage réussi dans notre réel social actuel : on s'aperçoit lors d'une enquête qu'un autre déterminant de la réussite est l'engagement dans la relation à autrui, y compris et surtout le «pas comme moi, nous », différent de ce qui est connu dans le réel social et familial de chaque élève (Berchoud, 1-11, 58 et s.). Parmi les élèves en difficultés scolaires : on peut distinguer en effet les conjoncturelles / durables; mais à quoi cela tient-il ou a-t-il tenu ? Les points essentiels sont :

- Une capacité à exister par soi-même et à rêver : en soi-même, et au dehors,

- Une ouverture à la rencontre, de soi à l'autre, et...les autres (adultes aidants),

- Un engagement dans l'aventure d'apprendre, à la fois intérieure, extérieure,

- Une capacité à ne rien tenir pour acquis qu'on ne l'ait vérifié, à (se) poser des questions,

- $\quad$ Et le goût de la découverte, non entravé, ni marginalisé.

Le déterminisme par le contexte socio-familial peut être ainsi mis en échec.... Ou alors se vérifier, mais cela par l'action d'un adulte au moins. C'est la petite S. qui va rater toute sa scolarité alors qu'elle avait bien commencé, et les adultes enseignants et encadrants le comprennent - mais trop tard - le jour où la maman lâche au psychologue scolaire « pourquoi irait-elle à l'école ? moi je n'y suis pas allée ! ». Mais il y a aussi les jumeaux C. et J., un garçon et une fille qui, placés dans deux classes différentes, vont finir par faire émerger leur individualité et réussir, alors que leur milieu n'est pas spécialement favorable ; et le petit M., dont le seul atout durant des années fut qu'il était excellent en vélo, a finit par transférer ses qualités sportives et mentales au domaine scolaire grâce à la rencontre de plusieurs adultes qu'il a pu entendre, écouter, suivre (58 et s.)

Alors, est-ce à dire que transgresser serait toujours un succès individuel contre une pression collective ? On l'a vu, ce n'est pas le cas, et en particulier, il faut considérer l'esprit au-delà de la lettre afin de ne pas ajouter une norme contraignante malgré les apparences, l'obligation d'être soi absolument et toujours (cf. supra, transgresser contre). Cependant cette tendance est fort présente dans la société actuelle, elle est même valorisée par les médias, 
notamment les médias dits sociaux, ce qui pousse à la recherche du sensationnel à tout prix, notamment les enfants et adolescents. Or apprendre, est-ce sensationnel ? Oui, en un sens, mais il faut le découvrir, il n'est pas donné d'emblée, en un clic, en une image.

Autrement dit, il faut reconsidérer la limite et le passage entre réel et fiction : Alice revient chez elle et sa vie continue, illuminée par son aventure; mais pour bien d'autres jeunes (et moins jeunes, la fiction ou le désir de fiction ont mangé leurs espoirs et leur vitalité pour les laisser démunis dans l'univers social. Il y aurait donc, de nos jours, une réflexion à mener sur la transgression, ses usages dans l'univers social étendu par les médias déterritorialisés et littéralement hors sol sociologique, familial, local, amical et scolaire ou professionnel.

\subsection{Usages et mésusages de la transgression}

Comment considérer la transgression instituée en nouvelle règle par les médias, une règle qui fait vendre car elle donne à voir ? Est-elle forcément mauvaise au motif qu'elle signerait le triomphe de «leurs Majestés les Médias» contre «Notre-Dame l'École» (Porcher) ? En réalité, et comme le dit Louis Porcher, tout est d'abord une question d'usage, même si la sociologie et le principe de réalité (économique) ensemble donnent les médias gagnants. La première réponse consiste pour un éducateur ou un enseignant à réutiliser les sketches, les vidéos, les clips dans l'apprentissage et l'éducation : ainsi des clips de Norman ou Cyprien, très prisés par les adolescents.

Mais que faire avec les sites à succès qui exploitent vraiment la bêtise, la le racisme, le sexisme, le mépris d'autrui, et le rire mauvais sur des situations dans lesquels sont attaqués les faibles (ou : placés en situation de faiblesse) ? C'est une vraie question, regardez par exemple le site à succès 9 gag.com devenu aussi grâce à son succès 9 gag TV. La réponse que je vois pour le moment est encore l'usage en classe : comme ce site et sa déclinaison télévisée existent uniquement en anglais, on peut faire travailler les élèves sur l'anglais parlé, son écoute, la prise de parole (souvent difficile pour les francisants) et, pour tous, sur les niveaux de langue, le lexique, etc. Le rire, même mauvais, permet de retenir l'attention, et de la focaliser sur un objet précis. Ensuite, pourquoi ne pas créer le sketch réplique, dans lequel les perdants sauraient répondre : voilà une chose très utile au double plan social et moral.

Prenons un autre exemple, moins voyant mais non moins inquiétant, de la puissance des médias dans la désintégration de l'être humain : lors d'une enquête sur le média radio, et plus particulièrement RFI, Radio France internationale, j'ai étudié le courrier des auditeurs de l'an 2000 (Berchoud, RFI), puis je suis revenue dix ans après sur cette enquête pour en apprécier les évolutions, et celles-ci sont quelque peu déprimantes. Ces évolutions tiennent à 
des décisions économiques et des choix d'orientations des dirigeants de cette radio, mais aussi à un contexte de montée en puissance des médis sociaux, particulièrement en Afrique.

Alors qu'en 2000, il était possible de voir dans le courrier des auditeursmajoritairement jeunes et scolaires ou universitaires - un mode d'expression de soi visant à l'élaboration de sa personnalité comme à l'utilisation de l'écriture scolaire en situation non scolaire, ce n'est quasiment plus le cas une dizaine d'années plus tard. Cela est dû d'abord au fort déclin des lettres papier, et à la montée en puissance des courriels - mais ceux-ci sont bien moins nombreux, plus brefs, et surtout ils ne manifestent pas d'élaboration particulière, cela étant sans doute dû au fait que l'accès à internet est collectif alors que le papier-crayon peut être utilisé de façon individuelle et, si on le veut, en retrait du groupe (Crinquand et Bravo 139-152).

Plus même, la radio RFI a créé en 2009100 blogs RFI, les Mondoblogs - voir rfi.fr mais pour y accéder il faut être sélectionné d'une part et, d'autre part, ces blogs sont collectifs (un groupe de jeunes dans telle ville, tel village). On assiste donc à un retour des conventions, à la perte du dire (présumé) individuel dans la lettre au profit de la poésie forcément collective et destinée à être vue plutôt qu'être lue par un destinataire pouvant être nommé. Il y avait en effet à RFI un service des relations auditeurs qui répondait aux lettres et chacun signait de son prénom ; aujourd'hui ce service est fortement réduit.

À la lumière de cette évolution sur un temps bref, on peut alors se demander si les médias d'aujourd'hui sont les seuls à avoir exploité le filon des tendances négatives de l'être humain. Faisons donc un bref détour historique. L'histoire des relations vécue avec autrui l'étranger en étant l'exemple le plus frappant - montre qu'il a fallu des siècles pour que l'étranger, le pas-come-nous, accède au statut d'être humain, et nous ne pouvons vraiment dire que c'est complètement du passé - complètement dépassé. Au Moyen-âge, comme Marco Polo dans Le devisement du monde (écrit à la fin du dix-huitième siècle) nous en est le témoin, si on s'intéresse à autrui, l'étranger, c'est pour ses techniques et ses merveilles - le sous-titre en est Le livre des merveilles - non pour dialoguer avec les hommes, vus comme totalement extérieurs à «notre » monde. À la Renaissance, viennent les temps des Grandes Découvertes... préparant les grandes colonisations et l'exploitation économique. Il faut dire qu'en ces temps l'esclavage existe déjà et sur bien des continents : généralement, ce sont les vaincus des guerres qui deviennent esclaves. L'autre, l'étranger sont donc vu comme objets à maîtriser, et seuls quelques écrivains et penseurs affirment leur humanité, par exemple Montaigne (chap. Des cannibales, I.31, Essais, 1572-1592). Puis viennent les siècles ouverts sur le voyage, et alors l'autre est exotique, tantôt bon sauvage et tantôt objet de curiosité, et à 
partir du dix-neuvième siècle, l'émergence de l'anthropologie posera la question de l'autre, question toujours ouverte : l'autre, alter ego, égal met différent; encore faut-il apprendre à vivre avec. Nous en sommes là, on peut se reporter au numéro spécial de la revue Le Français dans le monde - Recherches et applications « Culture, cultures...».

Nous pouvons dire pour clore cette seconde partie que la transgression est une conquête humaine, et non une régression; et que la tentation de la fiction, destinée à exclure l'autre du partage de notre humanité est toujours bien vive, car, n'est-ce pas l'autre qu'on torture, qu'on tue, qu'on exploite, il n'est pas vraiment des nôtres, de notre humanité... comme si l'humanité réelle ne pouvait tolérer le partage, l'engagement personnel, les incertitudes de l'altérité vécue.

\section{Pour conclure - La question de la transgression comme révélateur multiple}

Un bilan sur les pages qui précèdent amène à dire que la transgression dans les apprentissages doit être située, reliée, singulière et socialisée. En outre, si elle a un pied dans le rêve, elle ne peut durablement exister seulement par lui, car nous serions dans la fiction. Sur ce point, la montée en puissance des médias de proximité et des médias sociaux fait craindre un brouillage de la frontière réel - fiction, dans lequel autrui ne serait plus qu'une image destinée à d'autres buts que son existence propre. Les technologies et réseaux encouragent de fait la tendance au repli sur l'entre-soi, c'est-à- dire les pareils, en excluant les différences. Que devient alors la joie de la découverte - bien peu de choses, si tout est donné, su, validé a priori.

Des limites: posons ici que la transgression positive est celle qui cherche à comprendre et partager, celle qui cherche le savoir, au besoin jusqu'en Chine (vue d'ailleurs, évidemment). Mais il peut y avoir des pesanteurs (sociales, familiales...) qui freinent ou interdisent d'apprendre librement, de chercher le savoir. Prenons ici l'exemple vécu de certains apprenants illettrés adultes scolarisés dans une autre langue, qui n'arrivent pas à intégrer l'écrit en français, car certains gestes leur semblent impossibles, telles celui consistant à tracer les boucles de retour qui font changer le sens de l'écriture et viennent donc concurrencer d'autres graphies dont la toute première (voir Dautry, Les mots de l'espace entre expression et appropriation, 2009).

Des perspectives: même si des interdits culturels, sociaux, familiaux très forts subsistent, en particulier dans les apprentissages institutionnels, il apparaît possible de mener des projets amenant les élèves à réaliser «leur» transgression à leurs normes et craintes intériorisées, qu'il faut reconnaître pour mieux accompagner l'apprentissage. Ainsi, dans le 
perfectionnement en langue française pour les élèves de sections adaptées (SEGPA, IME, etc., élèves déficients ou en très grandes difficultés), quand les leçons de grammaire n'ont pas de sens car la langue demeure essentiellement orale, vécue, présente, partagée, il reste l'expérience vécue... et l'altruisme : le mémoire de Master d'Anne-Sophie Jeannin, fondé sur une expérimentation de plusieurs mois en classe, montre par exemple que si on propose à ces élèves de travailler à la rédaction d'un livret pour aider de jeunes élèves étrangers récemment arrivés en France et ne maîtrisant pas le français, alors l'enthousiasme est au rendez-vous, avec la motivation et le souci de l'autre. En aidant des enfants plus en difficultés qu'eux, ces élèves que le système scolaire a marginalisés (et ils le savent) retrouvent leur dignité, l'envie de faire, de dire, d'apprendre et de faire apprendre.

Alors, même si la notion de transgression créative, éducative et dialoguée s'ancre dans un modèle culturel de personnalité, d'éducation, de rapports sociaux, qui ne doit pas sous-estimer les autres modèles culturels ni occulter ses propres ratés (illettrisme, échecs scolaires et sociaux, migrants, quart-monde...), la transgression demeure une invite à penser à apprendre libres.

\section{Ouvrages cités}

Bart, Isabelle, dir. Regards actuels sur la pensée contemporaine: la pensée de Georges Simmel. Paris : Harmattan, 2009.

Bautier, Élisabeth. «Le rôle des pratiques des maîtres dans les difficultés scolaires des élèves ». Recherche et formation 51 (2006). http://rechercheformation.revues.org/497 Berchoud, Marie. RFI et ses auditeurs : «Chers émetteurs ... ». Paris : Harmattan, 2001.

---. 4-11 ans et difficultés à apprendre, Lyon : Chronique sociale, 2010.

---, Blandine Rui et Claire Mallet, dir. L'intime et l'apprendre. Berne : Lang, 2013.

Colas Blaise, Marion. Un enseignement moderne des langues vivantes étrangères pour les citoyens européens. Conférence Eurolingua, 16-18 avril 1998. Luxembourg: Wengler, 1998.

Crinquand, Sylvie et Paloma Bravo. L'intime à ses frontières. Bruxelles : EME, 2011.

Gourmelin-Berchoud, Marie. «Éthique et éducation à l'étranger». Le français dans le monde - Recherches et applications « Culture, cultures... ». Dir. Louis Porcher. 1999.

Hagège, Claude. L'enfant aux deux langues. Paris : Odile Jacob, 1996.

Jeannin, Anne-Sophie. U de Bourgogne, Dijon. Mémoire de Master EDH, 2013.

Meirieu, Philippe. «Apprentissage et transgression». Conférence, Entretiens Nathan. http://www.meirieu.com/ARTICLES, 2003. 
Neill, Alexander Sutherland. Libres enfants de Summerhill. Paris : Découverte, 1971. (Rééd 2004).

Porcher, Louis. Télévision, culture, éducation. Paris : Armand Colin, 1994.

\section{ANNEXES}

\section{1 - Images extraites de manuels scolaires d'hier}

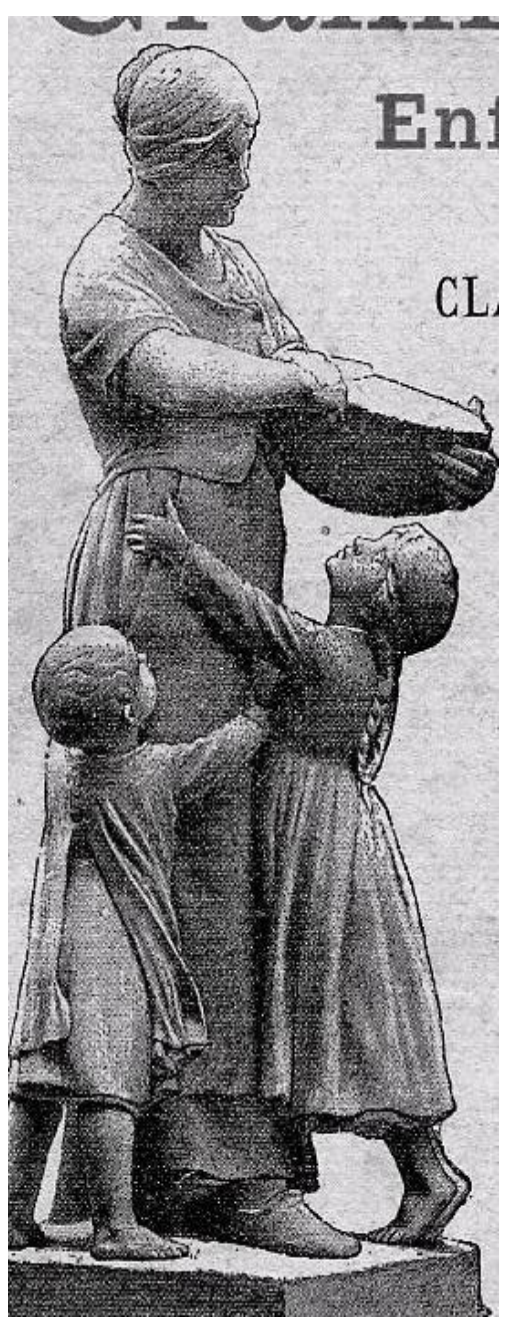

1910, Manuel de

Grammaire du français,

Paris. Image de couverture, puis image du premier chapitre (Avant le savoir / après) 


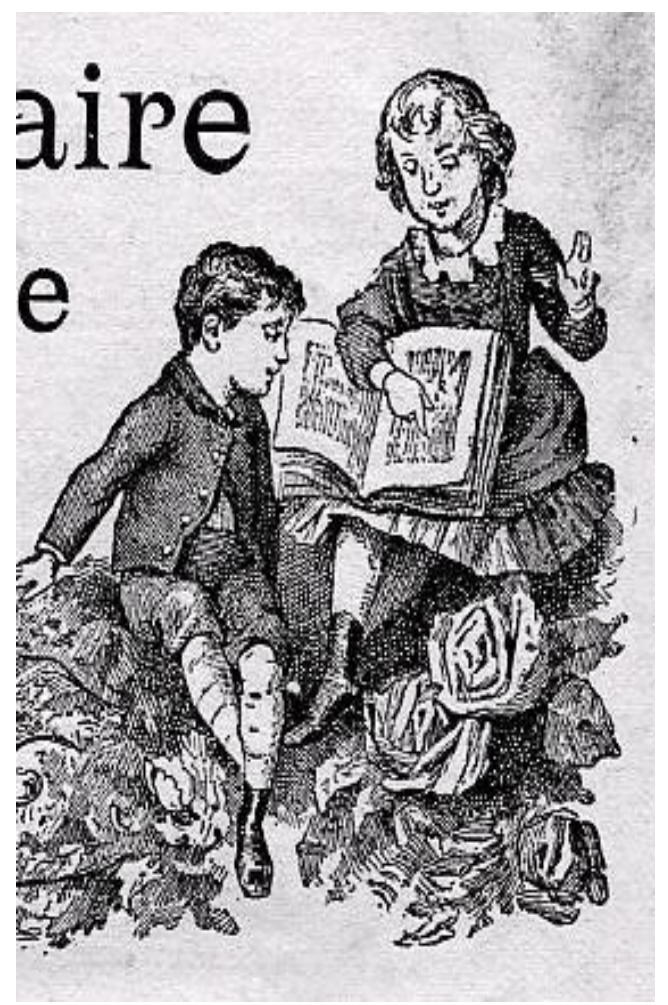

2 - Couverture d'un manuel français actuel (couverture disponible librement sur le net)

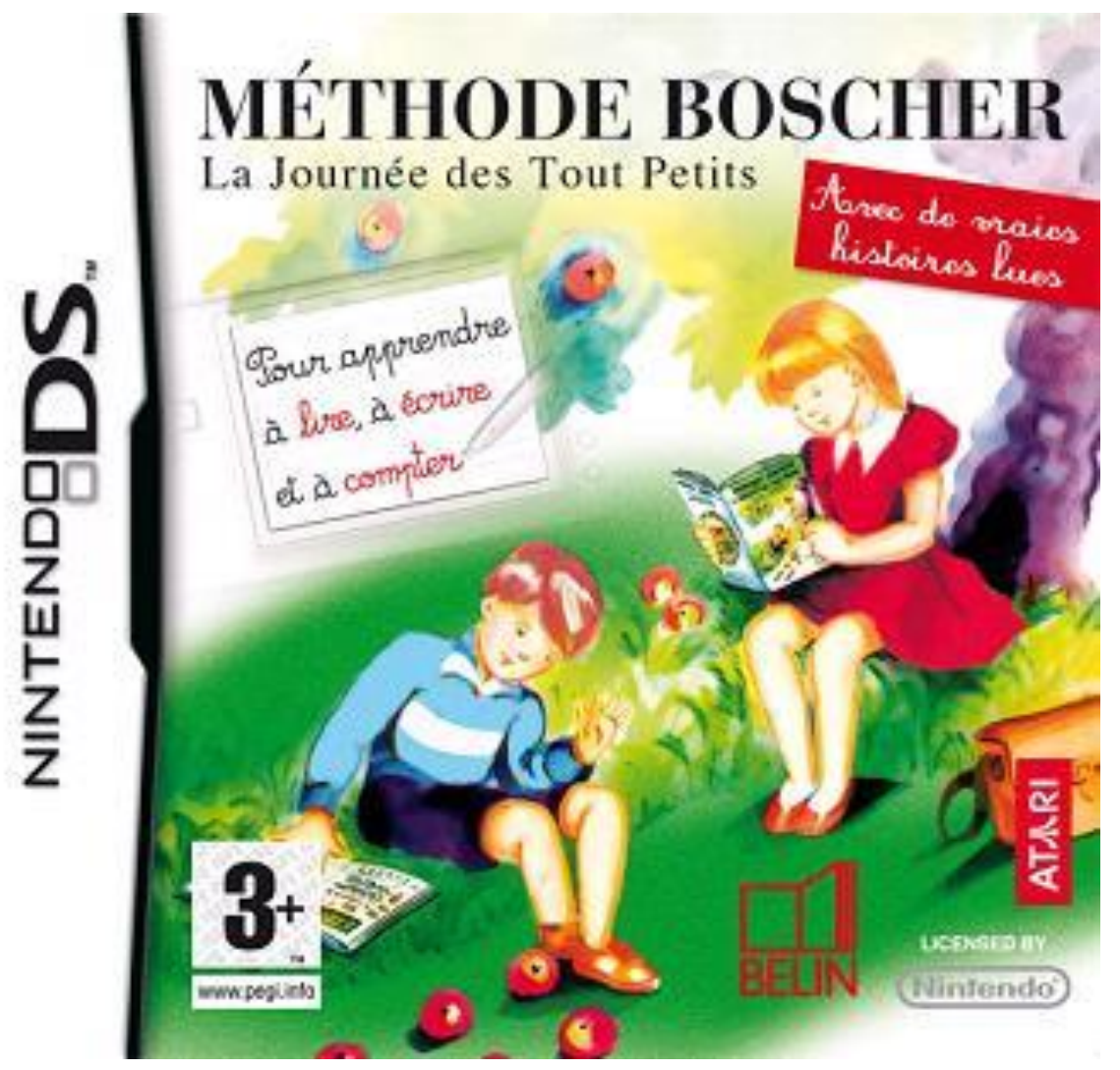

